\title{
Contrast-enhanced Ultrasound Assessment of Treatment Response in a Patient with Multifocal Hepatocellular Carcinoma Treated with Transarterial Chemo and Radioembolization
}

\author{
Esika Savsani \\ Thomas Jefferson University \\ M. Tantawi \\ Thomas Jefferson University \\ Corinne Wessner \\ Thomas Jefferson University
}

Philiputtis and additional works at: https://jdc.jefferson.edu/radiologyfp

I' mas Jefferson University

Part of the Radiology Commons

Andteillysshktritow how access to this document benefits you Thomas Jefferson University

\section{Recommended Citation}

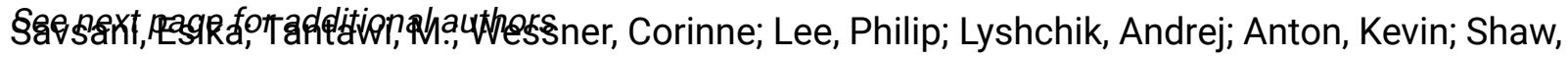
Colette; Liu, Ji-Bin; and Eisenbrey, John R., "Contrast-enhanced Ultrasound Assessment of Treatment Response in a Patient with Multifocal Hepatocellular Carcinoma Treated with Transarterial Chemo and Radioembolization" (2021). Department of Radiology Faculty Papers. Paper 116.

https://jdc.jefferson.edu/radiologyfp/116

This Article is brought to you for free and open access by the Jefferson Digital Commons. The Jefferson Digital Commons is a service of Thomas Jefferson University's Center for Teaching and Learning (CTL). The Commons is a showcase for Jefferson books and journals, peer-reviewed scholarly publications, unique historical collections from the University archives, and teaching tools. The Jefferson Digital Commons allows researchers and interested readers anywhere in the world to learn about and keep up to date with Jefferson scholarship. This article has been accepted for inclusion in Department of Radiology Faculty Papers by an authorized administrator of the Jefferson Digital Commons. For more information, please contact: JeffersonDigitalCommons@jefferson.edu. 


\section{Authors}

Esika Savsani, M. Tantawi, Corinne Wessner, Philip Lee, Andrej Lyshchik, Kevin Anton, Colette Shaw, Ji-Bin Liu, and John R. Eisenbrey 


\title{
Contrast-enhanced Ultrasound Assessment of Treatment Response in a Patient with Multifocal Hepatocellular Carcinoma Treated with Transarterial Chemo and Radioembolization
}

\author{
Esika Savsani ", Mohamed Tantawi, MD ${ }^{a}$, Corinne E. Wessner, MBA, RDMS, RVT ${ }^{a}$, Philip Lee, MD ${ }^{a}$, Andrej \\ Lyshchik, MD, PhD ${ }^{a}$, Kevin Anton, MD, PhD ${ }^{a}$, Colette M. Shaw, MD ${ }^{a}$, Ji-Bin Liu, MD ${ }^{a}$, John R. Eisenbrey, PhD ${ }^{a, *}$ \\ ${ }^{a}$ Department of Radiology, Thomas Jefferson University, Philadelphia, PA, USA
}

Received July 1, 2021; revision received July 22, 2021; accepted July 24, 2021

\begin{abstract}
Minimally invasive locoregional therapies have become important treatment options for patients with intermediate or late-stage hepatocellular carcinoma (HCC) who are ineligible for surgical resection or liver transplantation. Imaging modalities are essential for procedural guidance and for assessing treatment response thereafter. We report a unique finding of a patient with multifocal HCC treated with transarterial radioembolization (TARE) with yttrium-90 (Y90) and transarterial chemoembolization (TACE). We compared contrast-enhanced ultrasound (CEUS) with contrast-enhanced magnetic resonance imaging (CE-MRI) in the evaluation of treatment response to demonstrate advantages of CEUS imaging technique and its early detection of viable tumor.
\end{abstract}

Key words: Contrast-enhanced ultrasound; Hepatocellular carcinoma; Transarterial radioembolization; Transarterial chemoembolization; Treatment response

Advanced Ultrasound in Diagnosis and Therapy 2021; 03: 254-257

DOI: 10.37015/AUDT.2021.210018

$\Lambda$ lthough hepatocellular carcinoma (HCC) can be treated in its early stages with surgical resection, the disease is often discovered in the intermediate or late stage, rendering patients ineligible for resection. Thus, minimally invasive locoregional therapies have become an alternative treatment for these patients. Locoregional therapies can be curative, palliative, or downstage disease progression, and can also serve as a bridge to transplantation. Contrast-enhanced magnetic resonance imaging (CE-MRI) or contrastenhanced computed tomography (CE-CT) has been used as a reference standard for monitoring $\mathrm{HCC}$ treatment response to locoregional therapy. Contrast-enhanced ultrasound (CEUS) is another useful imaging technique used to identify viable tumor and treatment efficacy. Here we report a case of a patient with multifocal HCC treated with transarterial radioembolization (TARE) with yttrium-90 (Y90) and transarterial chemoembolization (TACE). We compared CEUS with CE-MRI in the evaluation of treatment response to demonstrate advantages of CEUS imaging technique and its early detection of viable tumor.

\section{Case Report}

A 58 year-old male patient with a history of hypertension, chronic kidney disease, coronary artery disease, and idiopathic cirrhosis presents with an incidental liver lesion found upon evaluation during an unrelated admission. The patient denies smoking, illicit drug use, or heavy alcohol use. CE-MRI demonstrated an arterially-enhancing, diffusion-restricting segment 6/7 liver mass, with washout in the delayed phase. The mass,

\footnotetext{
* Corresponding author: Thomas Jefferson University, 132 South 10th Street, 796E Main Building, Philadelphia, PA 19107, USA e-mail: John.eisenbrey@jefferson.edu unrestricted use, distribution and reproduction in any medium provided that the original work is properly attributed.
} 
measuring $6.8 \mathrm{~cm} \times 5.2 \mathrm{~cm}$, was consistent with $\mathrm{HCC}$ with a LIRADS-5 classification (Fig. 1). Laboratory examinations closest to diagnosis of $\mathrm{HCC}$ are as follows - Blood examination: WBC $9.2 \times 10^{3} / \mu \mathrm{L}, \mathrm{RBC} 4.24 \times$ $10^{3} / \mu \mathrm{L}, \mathrm{Hgb} 13.9 \mathrm{~g} / \mathrm{dL}$, Hct $41.2 \%$, Platelet $234 \times 10^{3} /$ $\mu \mathrm{L}$. Liver function: ALP $154 \mathrm{U} / \mathrm{L}$, AST $36 \mathrm{U} / \mathrm{L}$, ALT $20 \mathrm{U} / \mathrm{L}$, total bilirubin $0.5 \mathrm{mg} / \mathrm{dL}$, albumin $3.5 \mathrm{~g} / \mathrm{dL}$, AFP $321.5 \mathrm{ng} / \mathrm{mL}$ (AFP was first reported one year after diagnosis of HCC), INR 1.0, PTT 36sec. Child-Pugh classification was A with score of 6 points and BCLC was Stage B.

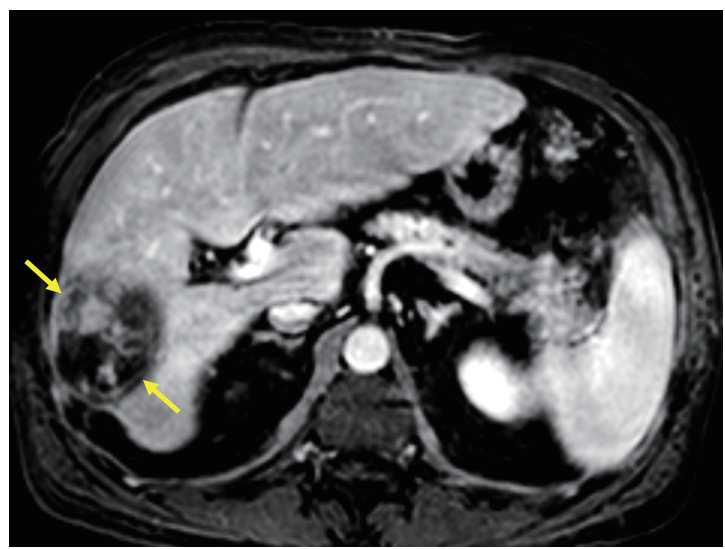

Figure 1 An axial T1 baseline CE-MRI of the early arterial phase demonstrated arterial phase hyperenhancement of the tumor (arrows) located at segment $6 / 7$ of the liver consistent with LIRADS-5 HCC.

The patient underwent Y90 radioembolization (Therasphere; BTG International, London, UK) to the segment 6/7 HCC (Fig. 2, Fig. 3). Five months post-Y90, the treated lesion was determined to be nonviable through routine clinical standard of care CE-MRI imaging (Fig. 4a). An incidental additional lesion was diagnosed as a $1.5 \mathrm{~cm}$ LIRADS-4 in segment $4 \mathrm{~B}$ (Fig. $4 \mathrm{~b})$. The patient underwent TACE for this segment $4 \mathrm{~B}$ lesion 6 months after the initial Y90 treatment. CEUS imaging was performed using a Logiq E9 scanner with a curved-array C1-6 probe (GE Healthcare, Milwaukee, WI) with a $0.2 \mathrm{~mL}$ bolus injection of Definity (Lantheus Medical Imaging, North Billerica, MA). CEUS conducted 2 weeks post-TACE, or 6 months post-Y90, revealed no viability in the segment $4 \mathrm{~B}$ lesion postTACE (Fig. 5a). Importantly though, CEUS data obtained during the same bolus injection demonstrated persistent vascularity within the segment $6 / 7$ lesion, indicative of viable tumor (Fig. 5b).

Contrary to the CEUS findings of viability, clinical standard of care CE-MRI imaging 8 months after Y90 assessed the segment 6/7 lesion as non-viable, with peripheral enhancement related to post-treatment effects (Fig. 6a). The segment 4B lesion post-TACE was also deemed non-viable on CE-MRI (Fig. 6b). Despite CEMRI findings of non-viability of both masses, the patient's AFP levels after TACE increased from $109.5 \mathrm{ng} /$ $\mathrm{mL}$ to $145.8 \mathrm{ng} / \mathrm{mL}$ in 2 months, indicative of potentially recurrent $\mathrm{HCC}$, and these levels continued to climb to $289 \mathrm{ng} / \mathrm{mL}$ over the next 3 months. A CE-MRI 10 months after $\mathrm{Y} 90$ assessed the segment $6 / 7$ post-Y90 lesion as equivocal with potential hyperenhancement and washout complicated with $\mathrm{T} 1$ hyperintensity (Fig. 7a), while the segment $4 \mathrm{~B}$ post-TACE lesion was again assessed as non-viable (Fig. 7b). The rising AFP levels, the continued non-viability of the segment $4 \mathrm{~B}$ lesion post-TACE, and the equivocal CE-MRI read for the segment 6/7 lesion post-Y90 suggests that the segment $6 / 7$ lesion is still viable; this finding is in agreement with the CEUS exam conducted 6 months post-Y90. Based on clinical and CEUS results, the segment $6 / 7 \mathrm{HCC}$ was re-evaluated for planned Y90 1 year after the initial treatment, and viability was confirmed during angiography (Fig. 8). The viable tumor was successfully retreated with Y90.

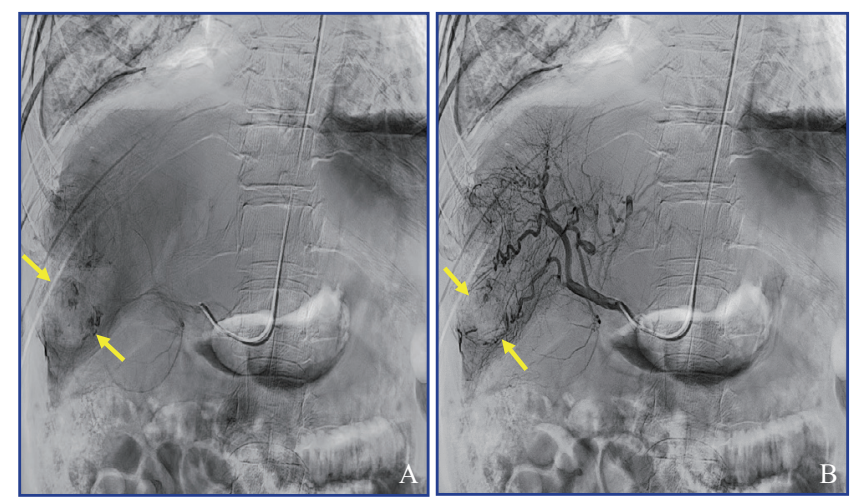

Figure 2 (A) A planning angiogram prior to Y90 pre-contrast injection showed the location of the tumor to be treated; (B) The post-contrast injection showed the tumor vascular supply to consider treatment mapping for the delivery of Y90 microspheres.

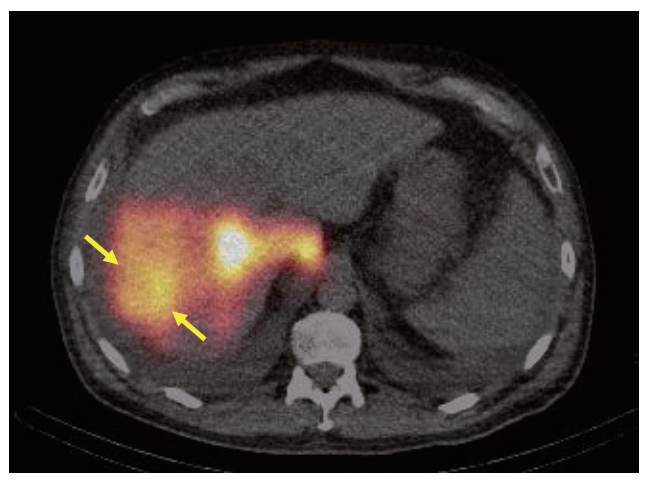

Figure 3 A SPECT exam prior to Y90 demonstrated localized delivery of the Tc99 radiotracer to the tumor (arrows) and a lung shunt fraction of $4.05 \%$ (quantified during post-processing), indicating that Y90 is appropriate to perform.

\section{Discussion}

Locoregional therapies play an essential role in the HCC treatment algorithm, and can be curative, palliative, or can downstage disease as a potential bridge to liver 
transplantation $[1,2]$. TARE with Y90 is a locoregional therapy which directs $20-40 \mu \mathrm{m}$ glass beads filled with radioisotope ${ }^{90} \mathrm{Y}$ via a catheter into the tumoral arterial network [3]. Alternatively, TACE is a catheter-directed locoregional therapy which delivers chemotherapy and an embolizing agent to the tumor [4]. In many cases, patients will have to undergo multiple locoregional treatments, which introduces the need for earlier detection of residual tumor $[5,6]$.

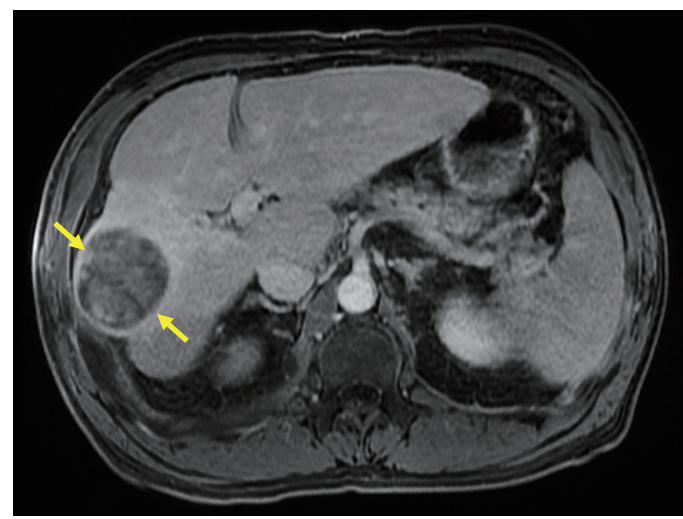

Figure 4 An axial T1 CE-MRI of the early arterial phase performed 5 months after Y90 showed a lack of arterial phase hyperenhancement within the tumor at segment $6 / 7$, suggesting that the tumor is non-viable.
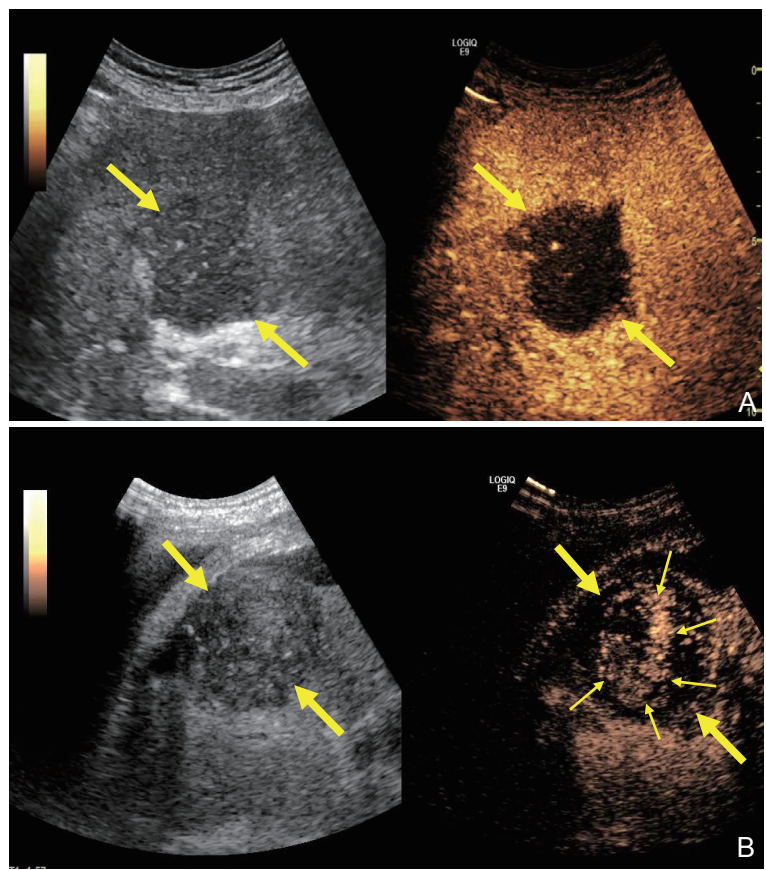

Figure 5 (A) The CEUS exam performed 2 weeks post-TACE (6 months post-Y90) revealed no enhancement within the treated lesion cavity (arrows), indicative of non-viability in the segment 4B tumor; (B) During the same bolus injection, the CEUS demonstrated vascularity (small arrows) within the tumor cavity (large arrows), indicative of tumor viability in the segment $6 / 7$ tumor.

CEUS has been studied as an alternative image modality to CE-MRI and CE-CT in the HCC patient population [7]. While studies have primarily focused

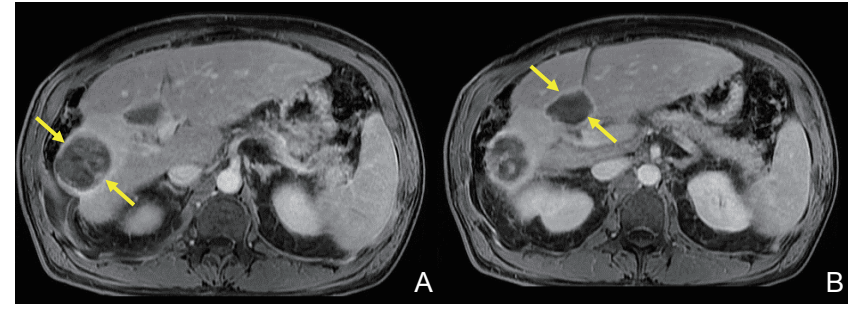

Figure 6 (A) An axial T1 CE-MRI of the early arterial phase performed 8 months after Y90 did not show arterial enhancement within the segment 6/7 Y90-treated cavity (arrows), and was called non-viable. This is contrary to the CEUS findings of viability; (B) The segment 4B TACEtreated cavity (arrows) was deemed non-viable, in accordance with the CEUS findings of non-viability.

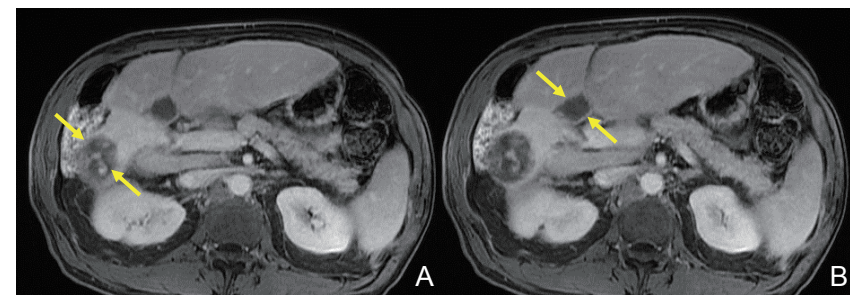

Figure 7 (A) An axial T1 CE-MRI of the early arterial phase performed 10 months after Y90 called the segment 6/7 tumor equivocal, with possible hyperenhancement (arrows). This is possibly in accordance with the CEUS findings of viability; (B) The segment 4B post-TACE lesion was again found to be non-viable (arrows), which is in accordance with the CEUS findings.
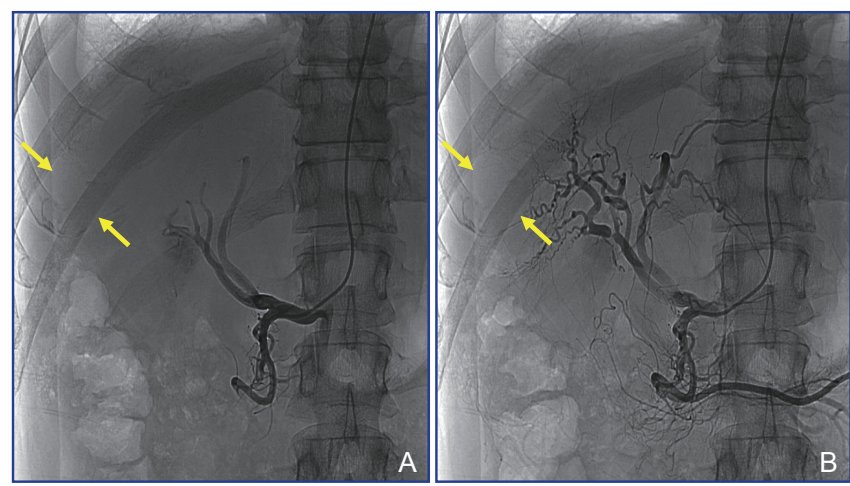

Figure 8 (A) A planning angiogram prior to the second scheduled Y90 to segment $6 / 7$ pre-contrast injection showed the location of the tumor to be treated; (B) The post-contrast injection demonstrated the tumor vasculature prior to the second Y90 treatment to map out the delivery of Y90 microspheres to the tumor.

on tracking response to TACE, similar approaches to Y90 have recently been investigated [8]. Gas-filled microbubbles surrounded by a lipid or protein shell, commonly known as ultrasound contrast agents (UCAs), are used for CEUS and injected intravenously [9]. UCAs are similar in size to erythrocytes and therefore can pass through the pulmonary-capillary bed; due to their stable shell surroundings, UCAs are not excreted [10]. CEUS has distinctive benefits over CE-MRI and CE-CT such as an absence of ionizing radiation and nephrotoxicity, high temporal resolution, lack of artifacts typically presented 
on CE-MRI or CE-CT shortly after locoregional therapy, and low cost $[11,12]$. Due to the high temporal resolution of CEUS, it is possible to image multiple locoregional therapy results simultaneously. CEUS has an increased sensitivity for detecting intramodular arterial blood flow as compared to other image modalities [13].

In summary, this case has shown the benefits of CEUS with accurate early detection of viable tumor after locoregional therapy. In this case, a CEUS exam 6 months after Y90 established residual tumor (Fig. 5b), while CE-MRI imaging 5 and 8 months after Y90 was unable to conclusively diagnose residual tumor (Fig. 4, Fig. 6a). Even 10 months after Y90, CE-MRI classified the lesion as equivocal, with less certainty than CEUS for viable tumor (Fig. 7a). These findings demonstrate the exceptional accuracy and flexibility of using CEUS to monitor $\mathrm{HCC}$ response to locoregional therapy.

\section{Acknowledgment}

This work was supported by NIH R01 CA238241. Equipment was provided by Siemens Healthineers.

\section{Conflict of Interest}

The authors have no conflict of interest to declare.

\section{References}

[1] Cardarelli-Leite L, Hadjivassiliou A, Klass D, Chung J, Ho SGF, Lim $\mathrm{HJ}$, et al. Current locoregional therapies and treatment strategies in hepatocellular carcinoma. Curr Oncol 2020; 27: s144-s151.

[2] Hibi T, Sugawara Y. Locoregional therapy as a bridge to liver transplantation for hepatocellular carcinoma within Milan criteria: from a transplant oncology viewpoint. Hepatobiliary Surg Nutr 2018;
7: 134-135.

[3] Singh P, Anil G. Yttrium-90 radioembolization of liver tumors: what do the images tell us? Cancer Imaging 2014; 13: 645-657.

[4] Raoul JL, Forner A, Bolondi L, Cheung TT, Kloeckner R, de Baere T. Updated use of TACE for hepatocellular carcinoma treatment: How and when to use it based on clinical evidence. Cancer Treat Rev 2019; 72: 28-36.

[5] Kirchner T, Marquardt S, Werncke T, Kirstein MM, Brunkhorst T, Wacker F, et al. Comparison of health-related quality of life after transarterial chemoembolization and transarterial radioembolization in patients with unresectable hepatocellular carcinoma. Abdom Radiol (NY) 2019; 44: 1554-1561.

[6] Piscaglia F, Ogasawara S. Patient selection for transarterial chemoembolization in hepatocellular carcinoma: importance of benefit/risk assessment. Liver Cancer 2018; 7: 104-119.

[7] Eisenbrey JR, Gabriel H, Savsani E, Lyshchik A. Contrast-enhanced ultrasound (CEUS) in HCC diagnosis and assessment of tumor response to locoregional therapies. Abdom Radiol (NY) 2021.

[8] Eisenbrey JR, Forsberg F, Wessner CE, Delaney LJ, Bradigan $\mathrm{K}$, Gummadi S, et al. US-triggered microbubble destruction for augmenting hepatocellular carcinoma response to transarterial radioembolization: a randomized pilot clinical trial. Radiology 2021; 298: 450-457.

[9] Dietrich CF, Averkiou M, Nielsen MB, Barr RG, Burns PN, Calliada $\mathrm{F}$, et al. How to perform contrast-enhanced ultrasound (CEUS). Ultrasound Int Open 2018; 4: E2-e15.

[10] Wink MH, Wijkstra H, De La Rosette JJ, Grimbergen CA Ultrasound imaging and contrast agents: a safe alternative to MRI? Minim Invasive Ther Allied Technol 2006; 15: 93-100.

[11] Pang EHT, Chan A, Ho SG, Harris AC. Contrast-enhanced ultrasound of the liver: optimizing technique and clinical applications. AJR Am J Roentgenol 2018; 210: 320-332.

[12] Xu E, Long Y, Li K, Zeng Q, Tan L, Luo L, et al. Comparison of CT/MRI-CEUS and US-CEUS fusion imaging techniques in the assessment of the thermal ablation of liver tumors. Int $J$ Hyperthermia 2019; 35: 159-167.

[13] Kudo M. Breakthrough imaging in hepatocellular carcinoma. Liver Cancer 2016; 5: 47-54. 